\title{
ON FUNCTIONS WITH INFINITELY MANY DERIVATIVES
}

\author{
ARTHUR ROSENTHAL
}

For a real function $f(x)$, analytic at $x=x_{0}$, the successive derivatives at $x=x_{0}$ can be chosen rather freely, but not in an entirely arbitrary manner since, if we set $a_{n}=(1 / n !) f^{(n)}\left(x_{0}\right)$, the power series

$$
\sum_{n=0}^{\infty} a_{n}\left(x-x_{0}\right)^{n}
$$

must have a positive radius $\rho$ of convergence. Of course, a necessary and sufficient condition which the $f^{(n)}\left(x_{0}\right)$ and the $a_{n}$ must satisfy is given by the Cauchy-Hadamard theorem; i.e., the sequence $\left|a_{n}\right|^{1 / n}$ must be bounded.

Hence the question arises in how general a manner the successive derivatives at $x=x_{0}$ can be assigned in the case of an infinitely often differentiable function $f(x)$. This question has already been answered by E. Borel [Ann. Ecole Norm. (3) vol. 12 (1895) pp. 35-44] ${ }^{1}$ who proved that to an infinitely often differentiable function $f(x)$ the values of the successive derivatives at $x=x_{0}$ can be assigned in an entirely arbitrary manner. In the following a new and simpler proof for this theorem is given, which uses quite a different method and yields an explicit procedure for computing the coefficients.

Without loss of generality we can set $x_{0}=0$. Let $b_{0}$ and $b_{m}$ $(m=1,2, \cdots)$ be the arbitrarily chosen finite, real values of $f(0)$ and $f^{(m)}(0)$, respectively. Now we define $f(x)$ as follows:

$$
f(x)=\sum_{\nu=0}^{\infty} c_{\nu} e^{-\left|c_{\nu}\right| \cdot \nu \mid x^{2} \cdot x^{\nu}}
$$

where the real numbers $c_{\nu}$ are to be determined by means of the given $b_{m}$.

Whatever the values of $c_{\nu}$ may be, the function $f(x)$ defined by (2) has infinitely many derivatives at every $x$ and $f^{(m)}(x)(m=1,2, \cdots)$ can be formed by differentiating (2) $m$ times term by term. For at the end of this proof, using some of the subsequent formulas, we shall show that the series (2) and the series obtained by differentiating (2) $m$ times term by term always converge absolutely and uniformly in any interval.

Presented to the Society, November 29, 1952; received by the editors October 21, 1952 and, in revised form, December 29, 1952.

${ }^{1}$ I wish to thank Professor Paul C. Rosenbloom for calling my attention to Borel's paper. 
Hence the value of $f^{(m)}(0)=b_{m}$ can be computed by differentiating the first $m+1$ terms of the series (2) $m$ times and then setting $x=0$. Thus $b_{m}$ can be represented by the $c_{0}, c_{1}, c_{2}, \cdots, c_{m}$ as follows. The $m$ th derivative of the $\nu$ th term of (2) has the form

$$
c_{\nu} \cdot e^{-\left|c_{\nu}\right| v \mid x^{2}} \cdot P_{m}^{y}(x) \quad(m=0,1,2, \cdots)
$$

where $P_{m}^{\nu}(x)$ is a polynomial of the degree $\nu+m$, satisfying the following formula of recursion:

$$
P_{m+1}^{\nu}(x)=\frac{d P_{m}^{\nu}(x)}{d x}-2\left|c_{\nu}\right| \nu ! x P_{m}^{\nu}(x)
$$

These polynomials are closely related to the Hermite polynomials $H_{m}(x)$. Indeed, for $\nu=0$ and $c_{\nu}=1 / 2$ (or $c_{\nu}=1$ according to some mathematicians), one has $P_{m}^{\prime}(x)=(-1)^{m} H_{m}(x)$. From (4) it follows that ${ }^{2}$

$$
P_{m}^{\nu}(x)=\sum_{s=0,(\nu \geq m-2 s)}^{m}\left(-2\left|c_{\nu}\right| \nu !\right)^{s} x^{p-m+2 s} k_{m, s}^{\nu}
$$

where in this sum only terms with $\nu \geqq m-2 s$ occur and where the constant factors $k_{m, s}^{\nu}$ (depending on $\nu$ ) can be successively determined by means of the formulas (always with $0 \leqq s \leqq m$ ):

$$
\begin{aligned}
k_{m+1, s}^{\nu} & =k_{m, s}^{\nu} \cdot(\nu-m+2 s)+k_{m, s-1}^{\nu} & \text { for } s=1,2, \cdots, m ; \\
k_{m, 0}^{\nu} & =\nu(\nu-1)(\nu-2) \cdots(\nu-m+1) & \text { for } m>0 ; \\
k_{m, m}^{\nu} & =1 \text { for } m \geqq 0 ; \quad k_{m, s}^{\nu}=0 & \text { for } \nu<m-2 s .
\end{aligned}
$$

Then from (2), (3), and (5) we obtain the desired representation of the $b_{m}$ by means of the $c_{0}, c_{1}, c_{2}, \cdots, c_{m}$ :

$$
b_{m}=\sum_{s=0}^{[m / 2]} c_{m-2 s} \cdot\left(-2\left|c_{m-2 s}\right| \cdot(m-2 s) !\right)^{s} k_{m, 8}^{m-2 s}
$$

where, as usual, $[m / 2]$ designates the greatest integer $\leqq m / 2$. Conversely if the $b_{m}$ are given, the $c_{m}$ can be successively determined by (7) in a unique manner (since in (7), for $s=0$, the coefficient of $c_{m}$, namely $k_{m, 0}^{m}=m !$, does not vanish).

To complete the proof we shall show that the series (2) and the series obtained by differentiating (2) $m$ times term by term converge absolutely and uniformly in any interval. For we shall show that,

\footnotetext{
${ }^{2}$ Here $\left(-2\left|c_{\nu}\right| \nu !\right)^{0}$ means 1 also if $c_{\nu}=0$; analogously in (7).
} 
whatever the $c_{\nu}$ may be, each such series converges at least as fast as the series

$$
\sum_{n=0}^{\infty} \frac{|x|^{n}}{(n+2) !}
$$

multiplied by a suitable constant.

According to (3) and (5) the $m$ th derivative of the $\nu$ th term of (2) (for $m=0,1,2, \cdots$ ) is the sum of at most $m+1$ terms of the form

$$
\begin{aligned}
c_{\nu} e^{-\left|c_{\nu}\right| \nu \mid x^{2}} \cdot\left(-2\left|c_{\nu}\right| \nu !\right)^{*} x^{\nu-m+2 \varepsilon} \cdot k_{m, s}^{\nu} \\
(s=0,1,2, \cdots, m ; \nu \geqq m-2 s) .
\end{aligned}
$$

The maximum of the absolute value of (9) for a variable $c_{\nu}$ and fixed $x(\neq 0), \nu, m, s$ is obtained for

$$
\left|c_{\nu}\right|=\frac{s+1}{\nu ! x^{2}}
$$

Hence this maximum equals

$$
2 \cdot\left(\frac{s+1}{e}\right)^{\circ+1} \cdot \frac{|x|^{\nu-m-2}}{\nu !} \cdot\left|k_{m, 8}^{\prime}\right| \text {. }
$$

But, by (6), $k_{m, s}^{v}$ considered as a function of $\nu$ for fixed $m$ and $s$ is a polynomial of $\nu$ of a degree at most $m$. Hence, setting $\nu-m-2=n$, we see from (10) that in fact each series obtained by differentiating (2) $m$ times term by term $(m=0,1,2, \cdots)$ converges at least as fast as the series ( 8 ) multiplied by a suitable constant.

PURdue UnIVERSITY 